\title{
Coherent generation of non-classical light on a chip via photon-induced tunnelling and blockade
}

\author{
ANDREI FARAON ${ }^{1 *}$, ILYA FUSHMAN ${ }^{1 *}$, DIRK ENGLUND ${ }^{1 *}$, NICK STOLTZ² ${ }^{2}$ PIERRE PETROFF² \\ AND JELENA VUČKOVIĆ ${ }^{1 \dagger}$
}

\author{
${ }^{1}$ E. L. Ginzton Laboratory, Stanford University, Stanford, California 94305, USA \\ ${ }^{2}$ Department of Electrical and Computer Engineering, University of California, Santa Barbara, California 93106, USA \\ *These authors contributed equally to this work \\ †e-mail: jela@stanford.edu
}

Quantum dots in photonic crystals are interesting because of their potential in quantum information processing ${ }^{1,2}$ and as a testbed for cavity quantum electrodynamics. Recent advances in controlling ${ }^{3,4}$ and coherent probing ${ }^{5,6}$ of such systems open the possibility of realizing quantum networks originally proposed for atomic systems ${ }^{7-9}$. Here, we demonstrate that non-classical states of light can be coherently generated using a quantum dot strongly coupled to a photonic crystal resonator ${ }^{10,11}$. We show that the capture of a single photon into the cavity affects the probability that a second photon is admitted. This probability drops when the probe is positioned at one of the two energy eigenstates corresponding to the vacuum Rabi splitting, a phenomenon known as photon blockade, the signature of which is photon antibunching ${ }^{12,13}$. In addition, we show that when the probe is positioned between the two eigenstates, the probability of admitting subsequent photons increases, resulting in photon bunching. We call this process photon-induced tunnelling. This system represents an ultimate limit for solid-state nonlinear optics at the single-photon level. Along with demonstrating the generation of non-classical photon states, we propose an implementation of a single-photon transistor ${ }^{14}$ in this system.

The optical system consists of a self-assembled InAs quantum dot with decay rate $\gamma / 2 \pi \approx 0.1 \mathrm{GHz}$ coupled to a three-hole defect cavity $^{15}$ in a two-dimensional GaAs photonic crystal, as described in ref. 5. The quantum-dot/cavity coupling rate $g / 2 \pi=16 \mathrm{GHz}$ equals the cavity field decay rate $\kappa / 2 \pi=16 \mathrm{GHz}$ (corresponding to a cavity quality factor $Q=10,000$ ), which puts the system in the strong coupling regime $e^{10,11}$. We first characterize the system in photoluminescence by pumping the structure above the GaAs bandgap. The photoluminescence scans in Fig. 1b show the anticrossing characteristic of strong coupling between the quantum dot and the cavity. Here, the quantum dot is tuned into resonance using local temperature tuning ${ }^{16}$ around an average temperature of $20 \mathrm{~K}$ maintained in a continuous He flow cryostat. To generate non-classical light, we coherently probe the system with linearly polarized laser beams (Fig. 1a) and observe the cross-polarized output, as described in our previous work ${ }^{5}$. The cross-polarized set-up enables us to separate the cavity-coupled signal from the direct probe reflection, which is essential for achieving large signal-to-noise ratios needed in autocorrelation measurements.

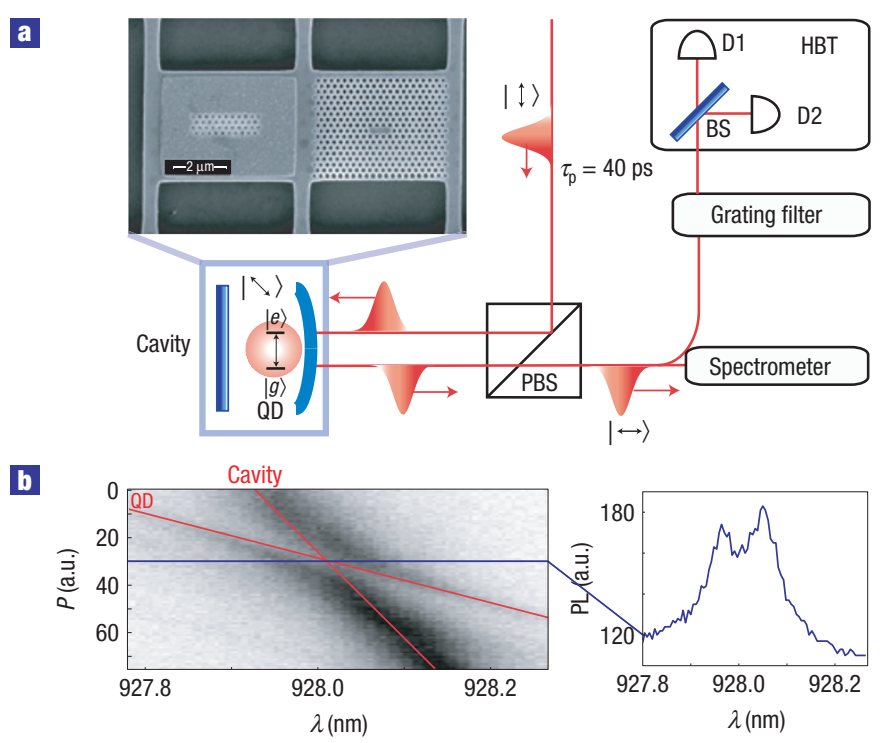

Figure 1 Schematic diagram of the experimental set-up. a, Laser pulses (40 ps FWHM) are reflected from a photonic crystal cavity that is linearly polarized at $45^{\circ}$ relative to the input polarization set by the polarizing beam splitter (PBS). The output light, observed in cross-polarization and carrying the cavity-coupled signal, is analysed using an HBT set-up that measures second-order correlation. The inset shows the suspended structure with the photonic crystal cavity and the metal pad for local temperature tuning ${ }^{16}$. BS: beam splitter; QD: quantum dot; D1 and D2: single-photon detectors. $\mathbf{b}$, Anticrossing observed in photoluminescence as the quantum dot is tuned into resonance with the cavity. The temperature tuning is done by linearly increasing the power $(P)$ of the heating laser ${ }^{16}$. The right panel shows the spectrum at the anticrossing point marked by the blue line. The red lines mark the cavity and quantum-dot resonance as if they were decoupled.

Our set-up is such that the measurement on the reflected port from this single-sided cavity is analogous to a transmission measurement in a Fabry-Perot arrangement. 
The energy eigenstates of a two-level system strongly coupled on resonance to an optical resonator are grouped into two-level manifolds denoted $|n, \pm\rangle$, with energies $\hbar \omega_{n, \pm}=\hbar\left(n \omega_{0} \pm g \sqrt{n}\right)$, where $n$ is the number of energy quanta in the system and $\omega_{0}$ is the bare-cavity frequency (Fig. 2a). The anharmonic energy-level spacing causes phenomena such as photon blockade ${ }^{12}$ or photon-induced tunnelling. To observe photon blockade, a coherent probe beam (frequency $\omega_{\mathrm{p}}$ ) tuned to $\omega_{1, \pm}=\omega_{0} \pm g$ is coupled to the cavity. This probe is resonant with the first-order manifold, but detuned from transitions to the second manifold, $\omega_{1 \rightarrow 2}=\omega_{0} \pm g(\sqrt{2}-1)$, as shown in Fig. 2a. Consequently, once a photon is coupled into the system, it suppresses the probability of coupling a second photon with the same frequency. As a result, the output field acquires sub-Poissonian statistics. In addition to photon blockade, photon-induced tunnelling is expected near the bare-cavity resonance $\left(\omega_{\mathrm{p}}-\omega_{0}=\Delta \omega_{\mathrm{p}} \rightarrow 0\right)$ : the absorption of a first photon enhances the absorption of subsequent photons owing to resonance with higher-order manifolds, so the output consists of 'photon bunches'. These phenomena are purely quantum effects that cannot be explained using semi-classical theories. These effects can be probed by measuring the second-order correlation function, $g^{(2)}(\tau)$. The signature of the photon blockade effect is the antibunching in $g^{(2)}(\tau)$ (that is, $g^{(2)}(0)$ is a local minimum, $\left.g^{(2)}(0)<g^{(2)}(\tau)\right)$, as recently demonstrated by Birnbaum et al. ${ }^{12}$ in an experiment with neutral atoms. In the case of photon-induced tunnelling, $g^{(2)}(0)$ is a local maximum.

In Fig. 2b, we simulate the theoretical output spectrum as a single-frequency probe beam is tuned through the cavity and indicate the resonance of the transitions $|0\rangle \rightarrow|1,+\rangle$ and $|1,+\rangle \rightarrow|2,+\rangle$. The simulated driving field injects an average cavity photon number $\langle n\rangle \sim 0.4$ when resonant with the polaritons in the first manifold, and slightly saturates the quantum dot. The expected second-order correlation function for our system is shown in Fig. 2c, where we plot the dependence of $g^{(2)}(0)$ on the detuning $\Delta \omega_{\mathrm{p}}$ of the probe from the anticrossing frequency $\omega_{0}$. As expected from the intuitive argument above, the simulation predicts photon bunching as $\Delta \omega_{\mathrm{p}} \rightarrow 0$. Photon blockade is evident in the antibunched region near $\Delta \omega_{\mathrm{p}} \sim \pm 1.5 \mathrm{~g}$ (Fig. $2 \mathrm{c}$, inset). The blockade does not occur at $\Delta \omega_{\mathrm{p}}= \pm g$ as previously explained because the linewidth of the eigenstates $(\sim \kappa)$ is comparable to the splitting of the manifolds $(\sim 2 g)$, which results in a significant overlap of the allowed transitions between consecutive manifolds. As $\Delta \omega_{\mathrm{p}} \rightarrow 0$, the probability of absorbing the first photon decreases. However, if a photon is absorbed, it enhances the probability of capturing subsequent photons, and produces a photon-bunched output.

We measure the time-dependent autocorrelation $g^{(2)}(\tau)$ using the Hanbury-Brown-Twiss (HBT) set-up shown in Fig. la and described in refs 17 and 18. The relevant features occur at timescales that correspond to the quantum-dot/cavity coupling rate $g$, enveloped by the coherence time ${ }^{19}$, as shown in Fig. $2 \mathrm{~d}$. The coherence time for our system is given by the cavity photon lifetime $1 / 2 \kappa \sim 5$ ps. Hence, the time-dependent features in $g^{(2)}(\tau)$ occur much faster than the 300 ps time-resolution of the single-photon counting modules in the HBT set-up. To resolve the relevant features, we sample the autocorrelation function by short pulses $\left(\Delta t_{\mathrm{FWHM}} \sim 40 \mathrm{ps}, \Delta \omega_{\mathrm{FWHM}} / 2 \pi \sim 12 \mathrm{GHz}\right.$, where FWHM stands for full-width at half-maximum) with a repetition rate of $12.5 \mathrm{~ns}$. This probe pulse duration represents a compromise between fast sampling and a linewidth that is narrow enough to resolve the relevant spectral features. In the remainder of this letter, we present the measurements of $g^{(2)}(\tau)$ for different detunings of the probe beam, denoted as $g^{(2)}\left(\tau, \Delta \omega_{\mathrm{p}} / g\right)$.

To observe photon blockade and photon-induced tunnelling, we measured the unnormalized second-order correlation function
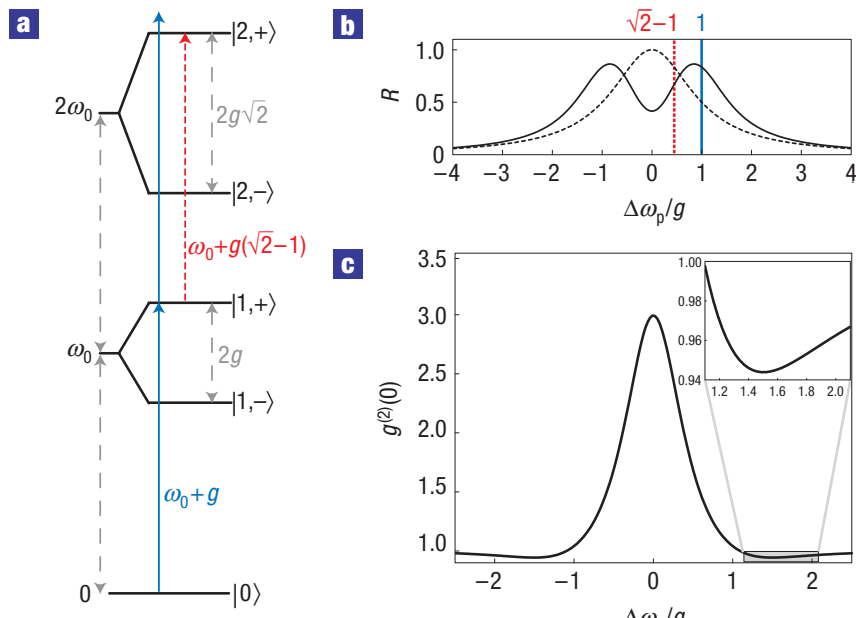

G



d

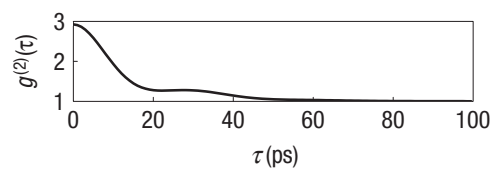

Figure 2 Theoretical analysis of the optical field reflected from the cavity. a, Energy diagram showing the first- and second-order manifolds of the strongly coupled cavity/quantum-dot system. The energy difference between consecutive manifolds is not constant, as shown by the blue and the red arrows. This anharmonic spacing of the levels causes phenomena such as photon blockade ${ }^{12}$ and photon-induced tunnelling. $\mathbf{b}$, Simulated output intensity for a probe beam frequency tuned through the strongly coupled cavity/quantum-dot system (solid line). The dotted line shows the bare-cavity reflectivity corresponding to the quantum dot in the dark state. The blue and red lines indicate the frequencies for the $|0\rangle \rightarrow|1,+\rangle$ and $|1,+\rangle \rightarrow|2,+\rangle$ transitions. c, Computed second-order correlation $g^{(2)}(0)$ for a coherent laser probe reflected from the cavity. The inset shows that photon blockade is expected when the probe detuning is $\Delta \omega_{\mathrm{p}} / g \sim 1.5$ because the absorption of a photon into $|1,+\rangle$ suppresses the probability of absorbing a second photon of the same energy for a transition to $|2,+\rangle$. The blockade does not occur exactly at $\Delta \omega_{p} / g=1$ because of the finite linewidth of the polaritons. As $\Delta \omega_{p} \rightarrow 0$, the absorption of a photon into the first manifold enhances the absorption probability into higher-order manifolds (photon-induced tunnelling) and results in a bunched output field. $\mathbf{d}$. Simulated time dependence of the second-order correlation for $\Delta \omega_{\mathrm{p}}=0$. The value for $g^{(2)}(\tau)$ drops rapidly for time delays greater than $\sim 5 \mathrm{ps}$, corresponding to the cavity photon lifetime.

at detunings $\Delta \omega_{\mathrm{p}} / g=1.5$ and $\Delta \omega_{\mathrm{p}} / g=0$ as shown in Fig. 3. The expected photon antibunching and bunching behaviour is clearly visible at zero time delay (Fig. 3b,d). The histograms also show bunching over timescales of hundreds of nanoseconds. This bunching is a purely classical effect that results from the Poissonian blinking of the quantum dot. As reported by Santori et al. ${ }^{20}$, such blinking is caused by quantum-dot transitions between a bright and a dark state, and results in bunching near $\tau=0$ that falls off with the mean switching rate. Our observations indicate that the blinking rates vary for different quantum dots. The quantum dot measured in this experiment spends $\sim 80 \%$ of the time in the bright state.

Photon blockade and photon-induced tunnelling are quantified by the normalized second-order correlation function $g^{(2)}\left(\tau, \Delta \omega_{\mathrm{p}} / g\right)$. Each peak in the histogram of Fig. 3 represents the unnormalized value of the second-order correlation averaged over the pulse duration of $40 \mathrm{ps}$. We express this time averaging by using the notation $\bar{g}^{(2)}\left(\tau, \Delta \omega_{\mathrm{p}} / g\right)$. The data are normalized such that 

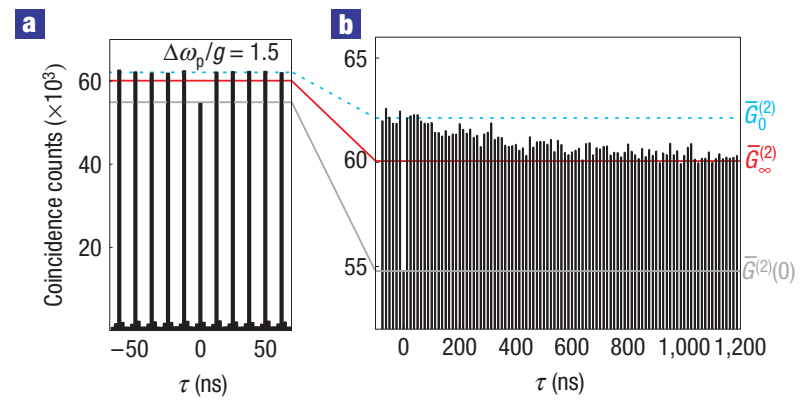

G

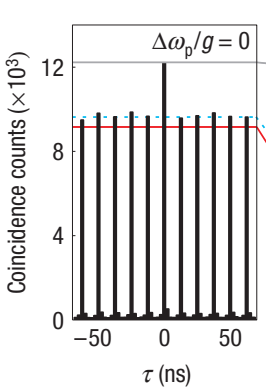

d

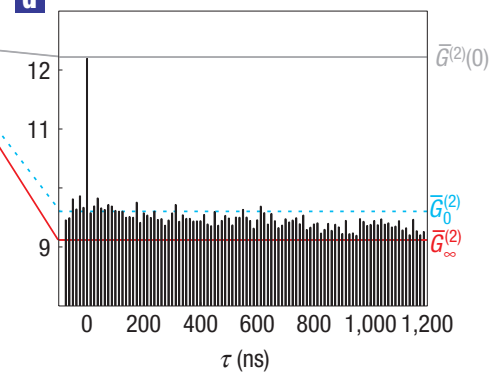

Figure 3 Measurement of the second-order correlation $\left(\bar{G}_{0}^{(2)}\left(\tau, \Delta \omega_{\mathrm{p}} / g\right)\right)$ function for coherent laser pulses reflected from the photonic crystal cavity with a strongly coupled quantum dot. a, Photon blockade, manifested here in the sub-Poissonian statistics, observed when the probe is detuned by $\Delta \omega_{\mathrm{p}} / g=1.5$. $\mathbf{b}$, The same data set as in a plotted for longer time delays. Along with the antibunching at $\tau=0$, bunching due to quantum-dot blinking can be observed over timescales of hundreds of nanoseconds. The red solid line marks the normalization level $\bar{G}_{\infty}^{(2)}$ for the correlation function. The blue dashed line indicates the classical bunching caused by quantum-dot blinking. The second-order correlation was computed as $\bar{g}^{(2)}(0,1.5)=\bar{G}^{(2)}(0) / \bar{G}_{\infty}^{(2)}=0.912$. c. Photon-induced tunnelling is observed when the laser pulse is tuned to the anticrossing point $\Delta \omega_{p} / g=0$. d, The same data set as in $\mathbf{c}$ plotted for longer time delays to make the classical blinking effects more visible. For this data set, the normalized second-order correlation was $\bar{g}^{(2)}(0,0)=1.37$.

$\bar{g}^{(2)}\left(\tau \rightarrow \infty, \Delta \omega_{\mathrm{p}} / g\right)=1$. We stress that $\bar{g}^{(2)}\left(\tau, \Delta \omega_{\mathrm{p}} / g\right)$ captures both the quantum and classical nature (blinking) of the output field. To find the normalization constant $\bar{G}_{\bar{G}}^{(2)}$, we fit the histogram with the function $\bar{G}^{(2)}\left(m T_{0}\right) \overline{\bar{G}(2)}\left(\bar{G}_{0}^{(2)}-\bar{G}_{\infty}^{(2)}\right) \exp \left[-m T_{0} / T\right]+\bar{G}_{\infty}^{(2)}$ for $m \geq 1$. The quantity $\bar{G}^{(2)}\left(m T_{0}\right)$ represents the number of counts at time $m T_{0}$, where $m$ indexes the peak number with $m=0$ corresponding to $\tau=0$ and $T_{0}=12.5 \mathrm{~ns}$ is the pulse repetition period. The normalized second-order correlation at $\tau=0$ is $\bar{g}^{(2)}\left(0, \Delta \omega_{\mathrm{p}} / g\right)=\bar{G}^{(2)}(0) / \bar{G}_{\infty}^{(2)}$ (see Fig. 3 for details). In the case of photon blockade, $\bar{g}^{(2)}(0,1.5)=0.912 \pm 0.005$, showing the antibunched quantum nature of the system. For photoninduced tunnelling, $\bar{g}^{(2)}(0,0)=1.37 \pm 0.02$ (Fig. 3d), which indicates bunching.

There are several factors that account for the difference between the theoretically predicted (Fig. $2 \mathrm{c}$ ) and measured values for $\bar{g}^{(2)}\left(0, \Delta \omega_{\mathrm{p}} / g\right)$ : background due to imperfect extinction of the cross-polarized experimental set-up (signal-to-noise ratio $\sim 6: 1$ ), quantum-dot blinking and finite bandwidth of the probe that affects the spectral resolution. Both the background and the output signal when the quantum dot is in the dark state result in a flat second-order correlation with $\bar{g}^{(2)}\left(\tau, \Delta \omega_{\mathrm{p}} / g\right)=1$ (coherent light). With the quantum dot in the dark state, the cavity reflectivity becomes that of an empty cavity, as shown by the dashed line in Fig. 2b. Near $\Delta \omega_{\mathrm{p}} / g=0$, the empty cavity (quantum dot in dark

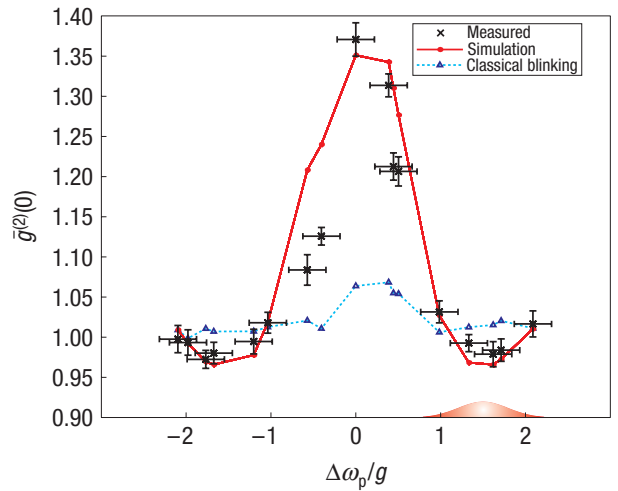

Figure 4 Measured normalized second-order correlation function $\bar{g}_{0}^{(2)}\left(0, \Delta \omega_{\mathrm{p}} / g\right)$ for different detunings between the probe and the anticrossing frequency. As the probe is tuned through the resonance of the quantum-dot/cavity system, the output field shows either antibunched or bunched behaviour as it transitions from the photon blockade regime to the photon-induced tunnelling regime. For comparison, we also plot the quantity $\bar{g}_{\text {blinking }}^{(2)}=\bar{G}_{0}^{(2)} / \bar{G}_{\infty}^{(2)}$, which corresponds to the bunching caused by quantum-dot blinking (blue dashed line). The red solid line shows the expected values of $\bar{g}_{0}^{(2)}\left(0, \Delta \omega_{\mathrm{p}} / g\right)$ determined through a simulation that takes into account the finite pulse width of the probe, quantum-dot blinking and background due to the imperfect extinction of the cross-polarized set-up. In the bottom right corner, we show the relative width of the pulsed laser probe. The vertical error bars are computed from the uncertainties in the fit of the histogram data sets. The horizontal error bars are given by the uncertainty in the measurement of the laser wavelength.

state) has high transmission, so the observed signal has a large coherent-state component. As a result, the region near $\Delta \omega_{\mathrm{p}} / g=0$ is expected to show the largest deviation in $g^{(2)}(0)$ compared with the ideal (non-blinking) dot in Fig. 2c. This deviation will bring the observed $g^{(2)}(0)$ closer to $g^{(2)}(0)=1$ of a coherent beam. At the blockade frequency $\left(\Delta \omega_{\mathrm{p}} / g \sim 1.5\right)$, the transmitted intensity in the dark state decreases relative to the bright-state intensity (Fig. 2b), and coherent light represents a smaller fraction of the collected signal.

We repeated the autocorrelation measurements for a large set of detunings to map the full spectrum of $\bar{g}^{(2)}\left(0, \Delta \omega_{\mathrm{p}} / g\right)$. The measurement of the full autocorrelation spectrum entails several challenges such as sample drift resulting in fluctuating coupling intensity into the cavity, and fluctuating temperature. To map the dependence of $\bar{g}^{(2)}\left(0, \Delta \omega_{\mathrm{p}} / g\right)$ on probe detuning, we maintained constant coupling into the cavity mode for the full duration of the experiment. In Fig. 4 we plot $\bar{g}^{(2)}\left(0, \Delta \omega_{\mathrm{p}} / g\right)$ for different detunings of the probe frequency. To emphasize the non-classicality of the signal, we plot in the same figure $\bar{g}_{\text {blinking }}^{(2)}\left(0, \Delta \omega_{\mathrm{p}} / g\right)$, the bunched second-order correlation resulting from quantum-dot blinking. For every autocorrelation measurement, the non-classical and classical contributions were easily distinguished by their greatly differing timescales as in Fig. 3c,d. The plots in Fig. 4 show the transition from the blockade regime $\left(\bar{g}^{(2)}<\bar{g}_{\text {blinking }}^{(2)}\right)$ to the photon-induced tunnelling regime $\bar{g}^{(2)}>\bar{g}_{\text {blinking }}^{(2)}$. The values for the classical bunching were obtained by taking the ratio $\bar{g}_{\text {blinking }}^{(2)}\left(0, \Delta \omega_{\mathrm{p}} / g\right)=\bar{G}_{0}^{(2)} / \bar{G}_{\infty}^{(2)}$ (see Fig. 3). As expected, $\bar{g}_{\text {blinking }}^{(2)}\left(0, \Delta \omega_{\mathrm{p}} / g\right)$ is higher as $\Delta \omega_{\mathrm{p}} / g \rightarrow 0$ because the intensity fluctuations due to blinking are largest at this detuning. While taking the data, we kept a constant probe power of $\sim 1.0 \mathrm{nW}$ before the objective lens $(\langle n\rangle \sim 0.4$ at the polariton frequency), and the coupling was re-optimized for every data point. The lowest value for $\bar{g}^{(2)}\left(0, \Delta \omega_{\mathrm{p}} / g\right)$ obtained in this 
a

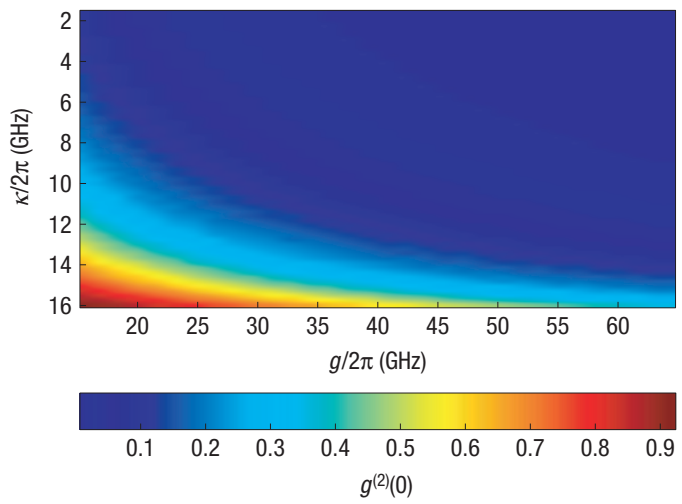

b

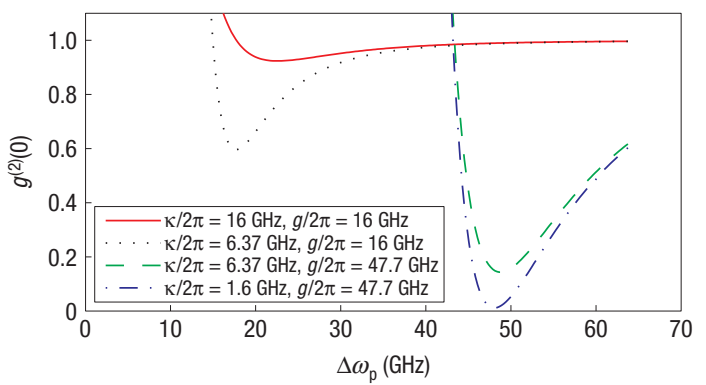

Figure 5 Expected photon blockade effect for various parameters of the strongly coupled system. a, Colour map plot for $g^{(2)}(0)$ obtained in the photon blockade regime for $1.6<\kappa / 2 \pi[\mathrm{GHz}]<16$ (that is, $10^{4}<Q<10^{5}$ ) and $16<g / 2 \pi[\mathrm{GHz}]<64$. b. Plot of $g^{(2)}(0)$ versus probe laser detuning for four relevant cases. $\kappa / 2 \pi=16 \mathrm{GHz}\left(Q=10^{4}\right), g / 2 \pi=16 \mathrm{GHz}$ (red line) corresponds to the system reported in this letter. $\kappa / 2 \pi=6.37 \mathrm{GHz}(Q=25,000), g / 2 \pi=16 \mathrm{GHz}$ (black dotted line) is for a system with coupling strength similar to the one in this letter but with the highest $Q$ measured in our laboratory. $\kappa / 2 \pi=6.37 \mathrm{GHz}$ $(Q=25,000), g / 2 \pi=48 \mathrm{GHz}$ (green dashed line) corresponds to the highest measured $Q$ and the highest achievable $g$ with this type of cavity and quantum dot. $\kappa / 2 \pi=1.6 \mathrm{GHz},\left(Q=10^{5}\right), g / 2 \pi=48 \mathrm{GHz}$ (blue dashed-dotted line) is for the highest achievable $g$ in this system and a quality factor four times higher than what has been currently achieved in GaAs photonic crystals at this wavelength.

data set is not as antibunched as the value $\bar{g}^{(2)}(0,1.5)=0.912$ reported in Fig. 3b, because we could not reproduce exactly the same coupling conditions. We found that the experimental data is well fitted by a numerical model that takes into account pulses of finite bandwidth, quantum-dot blinking and background from the imperfect extinction of the cross-polarized set-up (see Fig. 4 and the Methods section for details).

The experimental data in Fig. 4 show that, starting from a coherent state, the strongly coupled system enables control of the statistics of the output field from sub-Poissonian to super-Poissonian. Thus, by engineering the parameters of the system and by choosing the appropriate probe frequency, various non-classical states of light could be generated on demand. One of the most useful states is the single-photon state that has applications in quantum cryptography and distributed quantum networking. To achieve efficient single-photon sources based on photon blockade in strongly coupled solid-state systems, the quality factor $(Q)$ and the coupling strength $(g)$ need to be higher than those in our current work. In Fig. 5a, we show the expected second-order coherence $g^{(2)}(0)$ when operating in the blockade regime for the range of parameters $1.6<\kappa / 2 \pi[\mathrm{GHz}]<16$ (that is, $\left.10^{4}<Q<10^{5}\right)$ and $16<g / 2 \pi[\mathrm{GHz}]<64$. These estimations show that with $Q=25,000$ and $g / 2 \pi=48 \mathrm{GHz}$, values achievable in photonic crystals with InAs quantum $\operatorname{dots}^{21}$, the single-photon source should exhibit antibunching with $g^{(2)}(0) \sim 0.15$ (Fig. 5b). For even higher quality factors $\left(Q=10^{5}\right)$, almost complete antibunching is expected $\left(g^{(2)}(0) \sim 0.01\right)$. These simulations were carried out assuming continuous-wave weak excitation (average cavity photon number $\langle n\rangle \sim 0.01$ ) of the system.

Using the anharmonicity of the eigenenergy spacing in this system, a single-photon transistor ${ }^{14}$ could be implemented. In our transistor scheme, the frequency of the gate field is resonant with one of the polaritons in the first-order manifold, say $\omega_{0}+g$. A photon injected at $\omega_{0}+g$ increases the probability of absorbing photons that are resonant with the $|1,+\rangle \rightarrow|2,+\rangle$ transition at $\omega_{0}+g(\sqrt{2}-1)$. If the signal is tuned to this frequency, the presence of the gate field enhances the transmission of the signal field ${ }^{22}$. The photonic crystal architecture enables easy integration of such a single-photon transistor with photonic crystal waveguides ${ }^{23,24}$ so the single-photon switching is done directly on the chip. The most straightforward configuration would be a photonic crystal cavity butt-coupled in between two photonic crystal waveguides ${ }^{25}$. For a practical implementation, it is desirable that both the single-photon source and the single-photon transistor operate in pulsed mode, with one photon emitted (or switched) per pulse. The performance of the device depends on the coupling efficiencies in and out of the cavity, the bandwidth and the intensity of the pulse (a detailed analysis of the device performance will be the subject of further publications).

\section{METHODS}

\section{AUTOCORRELATION MEASUREMENT}

We scan several cavities until we find one that contains a strongly coupled quantum dot, as determined by the anticrossing behaviour in photoluminescence between the quantum dot and the cavity during temperature tuning. Then we direct the pulsed laser beam at the cavity and observe the reflected beam in cross-polarization. While tuning the local temperature with an extra heating beam, we adjust the probe beam coupling to optimize the quantum-dot-induced reflectivity drop, as described for the continuous-wave beam in ref. 5. Then we stop scanning and temperature-tune the quantum dot and cavity into resonance. With the pulsed probe beam at different detunings with respect to the anticrossing point, we measure the autocorrelation signal by passing the reflected probe through a grating filter (to remove stray light) followed by the HBT set-up. To limit sample drift, the alignment procedure is repeated for every data point in Fig. 4.

\section{DATA ANALYSIS}

The numerical model for the second-order coherence in Fig. 4 is based on numerical integration of the quantum master equation. A time-dependent driving term in the Hamiltonian represents the 40 ps excitation pulses. The intensity of the drive field matches the intensity used in the experiment, representing one-third of the quantum-dot saturation intensity. In our experiment, this intensity was $\sim 1 \mathrm{nW}$ for the incident beam, measured before the objective lens. The state of the quantum dot/cavity is time-evolved using a quantum Monte Carlo approach, which we based on the qotoolbox of ref. 26. The Hamiltonian is given by

$$
\begin{aligned}
H= & \left(\omega_{d}-\omega\right) \sigma_{+} \sigma_{-}+\left(\omega_{c}-\omega\right) a^{\dagger} a+i g\left(a^{\dagger} \sigma_{-}-\sigma_{+} a\right) \\
& +E(t)\left(a^{\dagger}+a\right),
\end{aligned}
$$

where the field $E(t)$ represents the time-dependent driving field (frequency $\omega$ ) of the cavity and is given by a sequence of Gaussian pulses. $a, a^{\dagger}$ denote the annihilation and creation operators of the cavity mode, and $\sigma_{+,-}$are the raising and lowering operators of the quantum dot. The quantum dot can emit into free space or into the cavity mode, which in turn leaks photons into the output channel at the loss rate $\omega / Q$. We then compute the autocorrelation on the output channel, as described in greater detail in ref. 27. The simulation also accounts for quantum-dot blinking and laser background. 
The full second-order coherence is calculated as a weighted sum of the different contributions,

$$
\begin{aligned}
G^{(2)}(\tau) & =\left\langle\mathbf{a}^{\dagger}(t) \mathbf{a}^{\dagger}(t+\tau) \mathbf{a}(t+\tau) \mathbf{a}(t) \rho\right\rangle \\
& =p_{\mathrm{B}} G_{\mathrm{B}}^{(2)}(\tau)+p_{\mathrm{BG}} G_{\mathrm{BG}}^{(2)}(\tau)+p_{\mathrm{D}} G_{\mathrm{D}}^{(2)}(\tau),
\end{aligned}
$$

where the autocorrelation function $G_{\mathrm{B}}^{(2)}(\tau)$ accounts for the quantumdot bright state, $G_{D}^{(2)}(\tau)$ for the quantum-dot dark state (calculated using $g \rightarrow 0$ ), $G_{\mathrm{BG}}^{(2)}(\tau)$ for background laser signal (a coherent state) and $p_{\mathrm{B}}, p_{\mathrm{D}}, p_{\mathrm{BG}}$ are the corresponding probabilities. The second-order correlation function for zero time delay is computed as $g^{(2)}(0)=G^{(2)}(0) / G^{(2)}(1) \cdot\left[G^{(2)}(1) / G^{(2)}(\infty)\right]_{\text {experiment }}$. Here, $G^{(2)}(1)$ is the autocorrelation of the nearest-neighbour peak to $\tau=0$ in the simulation. Owing to computational constraints, this is not evaluated at the actual pulse repetition time $\tau=12.5 \mathrm{~ns}$ but at $\tau=300 \mathrm{ps}$, a sufficient separation that amounts to nearly 60 coherence lengths. The factor $\left[G^{(2)}(1) / G^{(2)}(\infty)\right]_{\text {experiment }}$ is estimated from each autocorrelation measurement as $G^{(2)}(\tau=12.5 \mathrm{~ns}) / G^{(2)}(\infty)$.

Received 7 February 2008; accepted 22 August 2008; published 21 September 2008.

\section{References}

1. Loss, D. \& DiVincenzo, D. P. Quantum computation with quantum dots. Phys. Rev. A 57, 120-126 (1998)

2. Imamoglu, A. et al. Quantum information processing using quantum dot spins and cavity QED. Phys. Rev. Lett. 83, 4204-4207 (1999).

3. Atature, M. et al. Quantum-dot spin-state preparation with near-unity fidelity. Science $\mathbf{3 1 2}$ 551-553 (2006)

4. Berezovsky, J., Mikkelsen, M. H., Stoltz, N. G., Coldren, L. A. \& Awschalom, D. D. Picosecond coherent optical manipulation of a single electron spin in a quantum dot. Science 320, 349-352 (2008).

5. Englund, D. et al. Controlling cavity reflectivity with a single quantum dot. Nature $\mathbf{4 5 0}$, 857-861 (2007).

6. Srinivasan, K. \& Painter, O. Linear and nonlinear optical spectroscopy of a strongly coupled microdisk-quantum dot system. Nature 450, 862-866 (2007).

7. Cirac, J. I., Zoller, P., Kimble, H. J. \& Mabuchi, H. Quantum state transfer and entanglement distribution among distant nodes in a quantum network. Phys. Rev. Lett. 78, 3221-3224 (1997).

8. Felinto, D. et al. Conditional control of the quantum states of remote atomic memories for quantum networking. Nature Phys. 2, 844-848 (2006).

9. Moehring, D. L. et al. Entanglement of single-atom quantum bits at a distance. Nature $\mathbf{4 4 9}$ 68-71 (2007).
10. Hennessy, K. et al. Quantum nature of a strongly coupled single quantum dot-cavity system. Nature 445, 896-899 (2007).

11. Yoshie, T. et al. Vacuum Rabi splitting with a single quantum dot in a photonic crystal nanocavity. Nature 432, 200-203 (2004).

12. Birnbaum, K. M. et al. Photon blockade in an optical cavity with one trapped atom. Nature 436, 87-90 (2005).

13. Imamoglu, A., Schmidt, H., Woods, G. \& Deutsch, M. Strongly interacting photons in a nonlinear cavity. Phys. Rev. Lett. 79, 1467-1470 (1997).

14. Chang, D. E., Sorensen, A. S., Demler, E. A. \& Lukin, M. D. A single-photon transistor using nanoscale surface plasmons. Nature Phys. 3, 807-812 (2007).

15. Akahane, Y., Asano, T., Song, B.-S. \& Noda, S. High-Q photonic nanocavity in a two-dimensional photonic crystal. Nature 425, 944-947 (2003).

16. Faraon, A. et al. Local quantum dot tuning on photonic crystal chips. Appl. Phys. Lett. 90 213110 (2007).

17. Englund, D., Faraon, A., Zhang, B, Yamamoto, Y. \& Vučković, J. Generation and transfer of single photons on a photonic crystal chip. Opt. Express 15, 5550-5558 (2007).

18. Englund, D. et al. Controlling the spontaneous emission rate of single quantum dots in a two-dimensional photonic crystal. Phys. Rev. Lett. 95, 013904 (2005).

19. Carmichael, H., Brecha, R. J. \& Rice, P. R. Quantum interference and collapse of the wavefunction in cavity QED. Opt. Commun. 82, 73-79 (1991).

20. Santori, C. et al. Submicrosecond correlations in photoluminescence from InAs quantum dots. Phys. Rev. B 69, 205324 (2004).

21. Vučković, J., Englund, D., Fattal, D., Waks, E. \& Yamamoto, Y. Generation and manipulation of nonclassical light using photonic crystals. Physica E 32, 466-470 (2006).

22. Thompson, R. J., Turchette, Q. A., Carnal, O. \& Kimble, H. J. Nonlinear spectroscopy in the strong-coupling regime of cavity QED. Phys. Rev. A 57, 3084-3104 (1998).

23. Faraon, A., Waks, E., Englund, D., Fushman, I. \& Vuckovic, J. Efficient photonic crystal cavity-waveguide couplers. Appl. Phys. Lett. 90, 073102 (2007).

24. Noda, S., Chutinan, A. \& Imada, M. Trapping and emission of photons by a single defect in a photonic bandgap structure. Nature 407, 608-610 (2000).

25. Tanabe, T., Notomi, M., Kuramochi, E., Shinya, A. \& Taniyama, H. Trapping and delaying photons for one nanosecond in an ultrasmall high-Q photonic-crystal nanocavity. Nature Photon. 1 , 49-52 (2006).

26. Tan, S. M. A computational toolbox for quantum and atomic physics. J. Opt. B 1, 424-432 (1999).

27. Englund, D. Photonic Crystals for Quantum and Classical Information Processing. PhD. thesis, Stanford Univ. (2008).

\section{Acknowledgements}

Financial support was provided by the MURI Center for Photonic Quantum Information Systems (ARO/IARPA Program), ONR Young Investigator Award, I.F. was supported by the NDSEG fellowship and D.E. was supported by the NSF and NDSEG fellowships. Part of the work was carried out at the Stanford Nanofabrication Facility of NNIN supported by the National Science Foundation.

Author information

Reprints and permissions information is available online at http://npg.nature.com/reprintsandpermissions. Correspondence and requests for materials should be addressed to J.V. 